\title{
Ensemble Feature Extraction for Multi-Container Quality-Diversity Algorithms Supplementary Information
}

\section{Projection to the feature descriptors space of the hardcoded-4 case}

We want to assess how solutions found for all cases occupy the feature descriptors space of the reference hardcoded-4 case. To do so, we project these solutions into the feature space of the hardcoded-4 case. Figures 1 and 2 show representative instances of such projection, over only one experimental run. There are only very few overlaps between the FD spaces of the Feature Extraction cases with the hardcoded-4, suggesting that the solutions of the former occupies completely different behavioural niches compared to the solutions of the latter.

\section{Pairwise KL-Coverage}

We consider another way of comparing together the FD spaces, by using the KL-Coverage metric from [2], which was also used in the original AURORA paper [1], to check whether two FD spaces are similar to each other (possibly hinting at their representation capabilities). We extend the original definition to handle multi-containers scenarios:

$$
K L C=\sum_{c}^{C} \mathcal{D}_{K L}\left[E_{c} \| A_{c}\right]=\sum_{c}^{C} \sum_{i=1}^{10} E_{c}(i) \log \left(\frac{E_{c}(i)}{A_{c}(i)}\right)
$$

where $C$ is the set of all containers; and for a container $c: E_{c}$ and $A_{c}$ are respectively the reference and the compared distributions for container $c$. Lower KLC scores indicate more similar FD spaces.

Table 1 contains a pairwise comparison of the distribution of all solutions found by each case, by using the KL coverage metric.

\begin{tabular}{|c|c|c|c|c|c|c|c|c|c|c|c|c|}
\hline & 4 & is & & & ha & & & & & & & \\
\hline hardcoded-4 & $32 \pm 2.279$ & $37.529 \pm 5.204$ & $237.401 \pm 138.267$ & $67.478 \pm 58.851$ & $.213 \pm 9.204$ & $15.145 \pm 26.647$ & $110.498 \pm 60.944$ & $381.826 \pm 103.125$ & $33.672 \pm 24.755$ & $41.156 \pm 53.640$ & & 68.020 \\
\hline hardcoded-4- & $61 \pm 0.703$ & $56 \pm 4.013$ & $217.568 \pm 30.985$ & $60.626 \pm 55.809$ & $28.818 \pm 6.043$ & $13.950 \pm 24.685$ & $110.802 \pm 51.521$ & $2828+83181$ & $29.310 \pm 24.720$ & $41.447 \pm 49.777$ & $2 \pm 70.991$ & \\
\hline & $58 \pm 0.134$ & $8 \pm 1.109$ & $816 \pm 96.423$ & $333 \pm 52.903$ & $23.149 \pm 6.437$ & $15.965 \pm 28.100$ & $123.597 \pm 65.578$ & $4.563 \pm 107.843$ & $34.616 \pm 22.830$ & $84 \pm 48.329$ & $403 \pm 79$ & 1.481 \\
\hline & $7 \pm 0.375$ & +0 & \pm 0 & 54 & $17.760 \pm 5.380$ & $787 \pm 4$ & 571 & $2.045 \pm 10$ & $31.613 \pm 2$ & & $.783 \pm$ & \\
\hline qt-reco-4 & $71 \pm 0.406$ & $3.099 \pm 0.923$ & $267.139 \pm 89.560$ & $51.507 \pm 49.455$ & $18.478 \pm 5.756$ & $17.875 \pm 34.182$ & $135.863 \pm 71.914$ & $407.154 \pm 86.430$ & $31.978 \pm 21.130$ & $26.666 \pm 37.319$ & $60.443 \pm 58.063$ & $50.702 \pm 38.753$ \\
\hline & & & & 11 & & 4.528 & 074 & & & & & \\
\hline hardcoded-1 & $6 \pm 1.832$ & 24 & \pm 6 & \pm 5 & 11. & $15.867 \pm 27.894$ & 244 & 79 & 580 & 487 & 214 & .541 \\
\hline & \pm 0.609 & & 73 & 1 & & & & 59 & & & & 366 \\
\hline & & & & & & & & & & & & \\
\hline o-9-ns & \pm 0.218 & 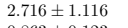 & \pm 86.652 & 80 & 90 & $=27.784$ & 28 & 0 & 340 & 33 & 134 & \pm 44.164 \\
\hline qt-re & & 3 & 36 & 12 & & $13.652 \pm 24.422$ & 30 & 395 & 30.88 & & 861 & \pm 36.077 \\
\hline qt-outputs-4-ns & 417 & & 232.5 & & .173 & 4.088 & & & & & & \pm 33.298 \\
\hline & 20 & & 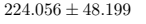 & 7 & 25. & 46 & & 40 & 92 & & 22 & \\
\hline 4-ns & \pm 0.214 & $928 \pm 0.254$ & $556 \pm 49.202$ & $29 \pm 60.668$ & $21.273 \pm 5.913$ & $.184 \pm 24.116$ & \pm 52.401 & 407 & $30.564 \pm$ & 65 & 67.4 & 8.2 \\
\hline & 233 & & \pm 55 & 77 & 232 & $13.675 \pm 2$ & $130.660 \pm 50.915$ & 415.3 & 29.847 & 30.25 & 68.87 & 6 \\
\hline
\end{tabular}

Table 1: Mean pairwise KL-coverage of all studied cases over 20 runs and using 10 bins per dimension: reference distributions are computed over the column cases and tested distributions over the row cases (so each pairwise score is averaged over $20 * 20=400$ KL-coverage scores). Lower scores mean distributions that are more similar. The numbers following the symbol \pm represent the standard deviation.

The results in Table 1 show that the distributions of solutions of pre-trained and online cases are larger than the ones of the human-designed cases (with low pairwise KL-coverage scores when human-designed cases are used to compute reference distributions). 

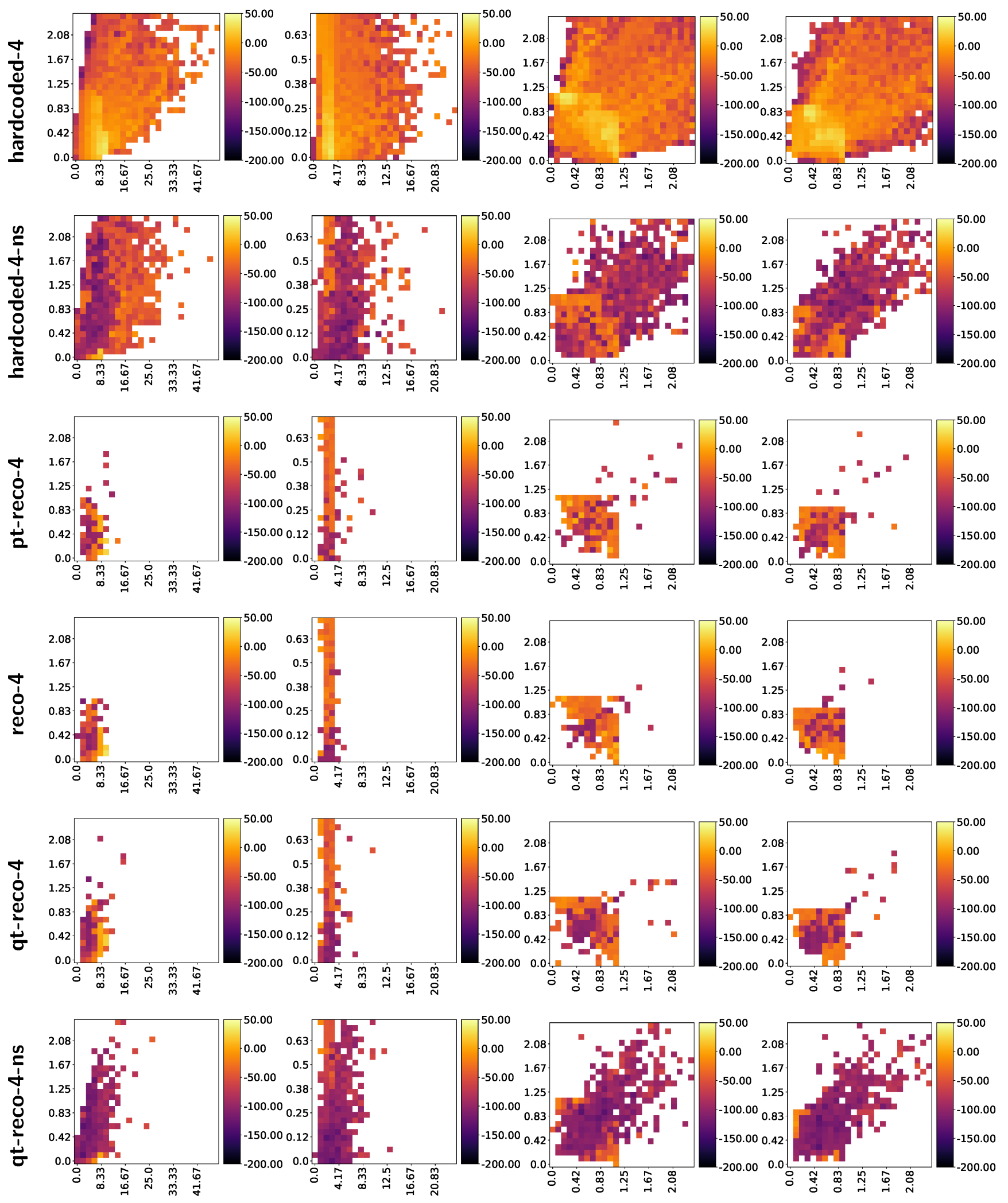

Figure 1: Representation instances (over one experimental run) of the projection of experimental cases into the feature descriptors space of case hardcoded-4. 



Figure 2: Representation instances (over one experimental run) of the projection of experimental cases into the feature descriptors space of case hardcoded-4. 


\section{References}

[1] A Cully. Autonomous skill discovery with quality-diversity and unsupervised descriptors. In Proceedings of the Genetic and Evolutionary Computation Conference, pages 81-89, 2019.

[2] A Péré, S Forestier, O Sigaud, and PY Oudeyer. Unsupervised learning of goal spaces for intrinsically motivated goal exploration. arXiv preprint arXiv:1803.00781, 2018. 